\title{
Mai1 Protein Acts Between Host Recognition of Pathogen Effectors and Mitogen-Activated Protein Kinase Signaling
}

\author{
Robyn Roberts, ${ }^{1}$ Sarah R. Hind, ${ }^{1}$ Kerry F. Pedley, ${ }^{1}$ Benjamin A. Diner, ${ }^{1}$ Matthew J. Szarzanowicz, ${ }^{1}$ \\ Dianiris Luciano-Rosario,, ${ }^{1}$ Bharat B. Majhi, ${ }^{2}$ Georgy Popov, ${ }^{2}$ Guido Sessa, ${ }^{2}$ Chang-Sik Oh, ${ }^{1,3}$ and \\ Gregory B. Martin ${ }^{1,3,4,+}$ \\ ${ }^{1}$ Boyce Thompson Institute for Plant Research, Ithaca, NY 14853, U.S.A. \\ ${ }^{2}$ School of Plant Sciences and Food Security, Tel-Aviv University, Tel-Aviv 69978, Israel \\ ${ }^{3}$ Department of Horticultural Biotechnology, College of Life Sciences, Kyung Hee University, Yongin 17104, Korea \\ ${ }^{4}$ Plant Pathology and Plant-Microbe Biology Section, School of Integrative Plant Science, Cornell University, Ithaca, NY 14853, \\ U.S.A. \\ Accepted 25 June 2019.
}

\begin{abstract}
The molecular mechanisms acting between host recognition of pathogen effectors by nucleotide-binding leucine-rich repeat receptor (NLR) proteins and mitogen-activated protein kinase (MAPK) signaling cascades are unknown. MAPKKK $\alpha$ $(\mathrm{M} 3 \mathrm{~K} \alpha)$ activates MAPK signaling leading to programmed cell death (PCD) associated with NLR-triggered immunity. We identified a tomato $\mathrm{M} 3 \mathrm{~K} \alpha$-interacting protein, SIMai1, that has 80\% amino acid identity with Arabidopsis brassinosteroid kinase 1 (AtBsk1). SIMai1 has a protein kinase domain and a $\mathrm{C}$ terminal tetratricopeptide repeat domain that interacts with the kinase domain of $\mathrm{M} 3 \mathrm{~K} \alpha$. Virus-induced gene silencing of Mail homologs in Nicotiana benthamiana increased susceptibility to Pseudomonas syringae and compromised PCD induced by four NLR proteins. PCD was restored by expression of a synthetic SlMail gene that resists silencing. Expression of AtBsk1 did not restore PCD in Mail-silenced plants, suggesting SIMai1 is functionally divergent from AtBsk1. PCD caused by overexpression of $\mathrm{M} 3 \mathrm{~K} \alpha$ or MKK2 was unaffected by Mail silencing, suggesting Mai1 acts upstream of these proteins. Coexpression of Mai1 with M3K $\alpha$ in leaves enhanced MAPK phosphorylation and accelerated PCD. These findings suggest
\end{abstract}

Robyn Roberts and Sarah R. Hind made equal contributions and are considered co-first authors.

Current address for S. R. Hind: Department of Crop Sciences, University of Illinois, Urbana, IL 61801, U.S.A.

Current address for K. F. Pedley: USDA-Agricultural Research Service, Foreign Disease-Weed Science Research Unit, Fort Detrick, MD 21702, U.S.A.

${ }^{\dagger}$ Corresponding author: G. B. Martin; gbm7@cornell.edu

Funding: This research was supported by the National Science Foundation (IOS-1451754; G. B. Martin), the USDA-Binational Agricultural Research and Development Fund (IS-4931-16C; G. B. Martin and G. Sessa), and by the National Research Foundation of Korea (NRF) grant funded by the Korea government (MSIT) (2018R1A5A1023599, SRC) to C.-S. Oh.

The $\boldsymbol{e}$-Xtra logo stands for "electronic extra" and indicates that 15 supplementary figures, two supplementary tables, and supplementary methods are published online.

The author(s) declare no conflict of interest.

๑ 2019 The American Phytopathological Society
Mai1 is a molecular link acting between host recognition of pathogens and MAPK signaling.

Keywords: bacterial speck disease, NLR-triggered immunity, plant immunity, Pseudomonas syringae, signal transduction, tomato

Plants use intracellular immune receptors to detect and respond to specific virulence (effector) proteins that pathogens translocate into the host cell as part of their infection process (Maekawa et al. 2011; Qi and Innes 2013). These receptors are typically members of the family of nucleotide-binding leucinerich repeat receptors (NLRs) and may have either a coiled-coil (CC) or a Toll-interleukin-1 receptor (TIR) domain (Jones et al. 2016; Takken and Goverse 2012). In some cases, additional NLRs promote the function of sensor NLRs, although their mechanisms are unknown (Dong et al. 2016; Wu et al. 2015, 2017). Nucleotide binding is thought to maintain NLRs in an equilibrium state with ATP hydrolysis shifting the NLR to an ADP-bound inactive state and detection of a pathogen effector shifting the NLR to the ATP-bound activated state (Zhang et al. 2017). Oligomerization of NLRs mediated by the CC or TIR domain is often essential for NLR activation (Gutierrez et al. 2010; Wang et al. 2019a and b). The molecular mechanisms that promote signaling upon NLR activation are not wellunderstood (Zhang et al. 2017). TIR-NLRs often rely on the EDS1 lipase-like protein, whereas CC-NLRs often rely on the NDR1 integrin-like protein, suggesting these two classes of NLRs might involve different early signaling partners (Aarts et al. 1998). Recently a CC-NLR, NRG1, has emerged as a candidate for an early TIR-NLR-specific signaling component (Brendolise et al. 2018; Castel et al. 2019; Qi et al. 2018; Wu et al. 2019).

The interaction of tomato and Nicotiana benthamiana with the bacterial pathogen Pseudomonas syringae pv. tomato is a model system for investigating the molecular basis of NLR activation and associated signaling (Ntoukakis et al. 2014; Pedley and Martin 2003). The CC-NLR Prf acts in concert with host protein kinases Pto and Fen to activate NLR-triggered immunity (NTI) (Gutierrez et al. 2010; Mucyn et al. 2009). Pto and Fen bind the $P$. syringae pv. tomato effectors AvrPto or AvrPtoB, possibly by acting as 'decoys' of the virulence targets of these effectors (Abramovitch and Martin 2005; Martin 2012; van der Hoorn and Kamoun 2008). Pto and Fen interact constitutively with an N-terminal domain of Prf, and the interaction 
contributes to the stabilization of both the NLR and Pto/Fen (Mucyn et al. 2006, 2009; Xiao et al. 2007). Pto and Fen can autophosphorylate and this activity appears to be required for changing the kinases into an active form but is not necessary for downstream signaling (Jia et al. 1997; Mathieu et al. 2014). Like other NLRs, Prf likely cycles between inactive (ADPbound) and active (ATP-bound) forms (Du et al. 2012). As with other NLR-mediated pathosystems, little is known about early signaling steps following Prf activation. Recently however, two NLRs, NRC2 and NRC3, and a cytoplasmic kinase, Epk1, of the GmPK6/AtMRK1 family have been shown to be required for Pto/Prf-associated PCD and resistance to P. syringae pv. tomato, although where exactly in this NTI pathway these proteins operate is unknown (Pombo et al. 2014; Wu et al. 2016, 2017).

Host responses activated by Pto/Prf are typical of NTI and include phosphorylation of mitogen-associated protein kinases (MAPKs) (Meng and Zhang 2013; Pedley and Martin 2005), transcriptional reprogramming (Jia and Martin 1999; Pombo et al. 2014), generation of reactive oxygen species (Chandra et al. 1996), and localized programmed cell death (PCD) (Coll et al. 2011; Du et al. 2012), which is thought to inhibit spread of the pathogen in host tissues. The role and mechanisms associated with MAPK signaling have been well-characterized in the Pto/Prf pathway. Virus-induced gene silencing (VIGS) of two tomato MAPKK genes (SlMKK1 and SlMKK2) and two MAPK genes (SIMPK2 or SIMPK3) compromised Pto/Prfmediated resistance and initially revealed a role for MAPK cascades in this pathway (Ekengren et al. 2003; Tena et al. 2001). NTI-associated MAPK signaling is also important in Arabidopsis and rice (Agrawal et al. 2003; Colcombet and Hirt 2008; Meng and Zhang 2013; Rasmussen et al. 2012; Tsuda et al. 2009). A subsequent VIGS screen in N. benthamiana, which tested the effect of silencing more than 2,400 randomly chosen cDNAs on NTI, identified one MAPKKK, M3K $\alpha$, as playing an important role in Pto/Prf-mediated immunity (del Pozo et al. 2004). Silencing of $M 3 K \alpha$ abolished PCD associated with Pto/Prf activation as well as cell death associated with P. syringae pv. tomato-related disease symptoms (del Pozo et al. 2004). In Pto/Prf-expressing leaves, transient expression of AvrPto or M3K $\alpha$ revealed that both increased the activity of SIMPK2 and SIMPK3 (del Pozo et al. 2004; Pedley and Martin 2004). M3K $\alpha$ is a member of group A2 in the MEKK (MAPK/ERK kinase kinase) family, and this group has since been found to contain other members that function in NTI (Hashimoto et al. 2012). Here, we describe the identification and characterization in $N$. benthamiana of a tomato $\mathrm{M} 3 \mathrm{~K} \alpha-$ interacting protein, Mai1, a receptor-like cytoplasmic kinase (RLCK), and present data suggesting it acts as a molecular link between early pathogen recognition events and MAPK signaling.

\section{RESULTS}

\section{SIMai1 interacts via its TPR domain}

with the kinase domain (KD) of SIM3K $\alpha$.

The tomato $\mathrm{M} 3 \mathrm{~K} \alpha(\mathrm{SIM} 3 \mathrm{~K} \alpha)$ protein was used as a bait in a yeast two-hybrid screen of a cDNA prey library generated from Rio Grande-PtoR tomato leaves inoculated with $P$. syringae pv. tomato (Fig. 1A) (Oh and Martin 2011; Zhou et al. 1995). From this screen, 18 clones were identified that contained sequences derived from the same gene, which was called Mail (M3K $\alpha$ interacting 1; Solyc04g082260). The tomato Mai1 (SlMai1) protein is a predicted RLCK with 497 amino acids. The most similar protein in Arabidopsis is BRASSINOSTEROIDSIGNALING KINASE 1 (AtBSK1) (Supplementary Fig. S1), which was originally identified as a substrate of the BRI brassinosteroid receptor and later implicated in immunity (Shi et al. 2013a and b; Tang et al. 2008; Yan et al. 2018). In rice, there are two proteins related to SIMai1, OsBSK1-1 and OsBSK1-2, with OsBSK1-2, also having a reported role in immunity (Wang et al. 2017). Subsequent assays showed that SIMai1 interacts with the SIM3K $\alpha$ KD and not with its N- or Cterminal domains (Fig. 1B). Immunoblotting showed that all proteins were expressed (Supplementary Fig. S2).

SIMai1 has residues in its $\mathrm{N}$ terminus that are predicted to be myristoylated or palmitoylated (i.e., MGCC), a central KD, and a region of tetratricopeptide repeats (TPR) in the $\mathrm{C}$ terminus (Fig. 1C). AtBSK1 was reported to be an active kinase in vitro, and an amino acid substitution in its ATP binding site (K104E) compromised its role in resistance to a fungal pathogen (Shi et al. 2013b). Like all BSK proteins, SIMai1 lacks specific

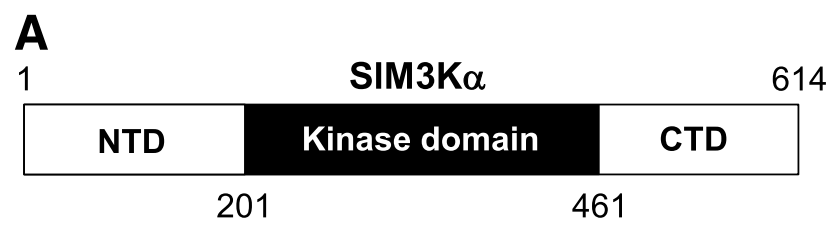

B

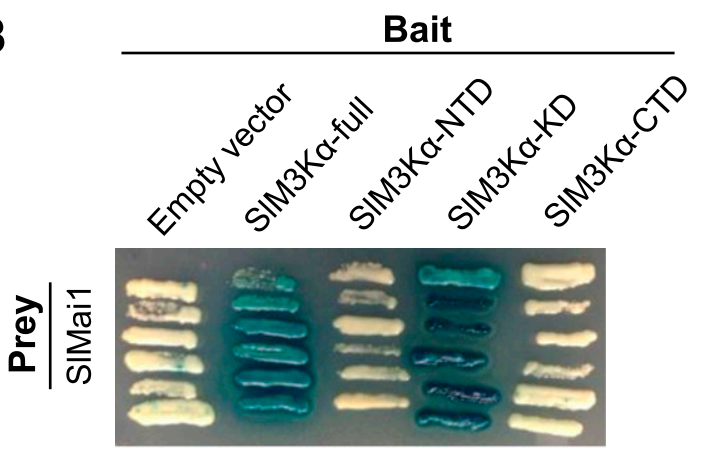

C

153

$53 \quad$ SIMai1

Kinase domain

TPR

MGCC

318384

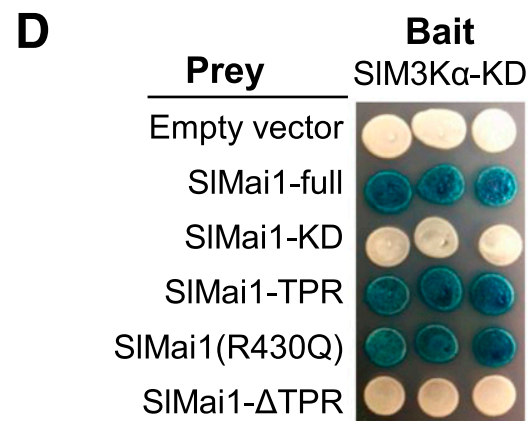

Fig. 1. Tomato Mai1 (SIMai1) interacts via its tetratricopeptide repeat (TPR) domain with the SIM3K $\alpha$ kinase domain (KD). A, Schematic of tomato $\mathrm{M} 3 \mathrm{~K} \alpha(\mathrm{SlM} 3 \mathrm{~K} \alpha)$ showing the $\mathrm{N}$ - and $\mathrm{C}$-terminal domains (NTD and $\mathrm{CTD}$ ) and the KD along with the amino acid coordinates. B, SIMai1 was tested in a yeast two-hybrid (Y2H) assay for interaction with SIM3Ka full-length and its NTD, KD, and CTD subdomains. C, Schematic of SIMail showing the KD, TPR domain, and myristoylation/palmitoylation motif (MGCC) along with amino acid coordinates. D, SIMai1, its KD and TPR subdomains, and the SIMai1(R430Q) variant were tested for interaction with SIM3K $\alpha-\mathrm{KD}$ in a yeast two-hybrid assay. For all $\mathrm{Y} 2 \mathrm{H}$ assays, SIMai1 was expressed as the prey protein fused to the activation domain in pJG4-5 and SIM3K $\alpha$ was expressed as the bait protein fused to the LexA DNA-binding domain in pEG202. Dark patches indicate a positive interaction. 

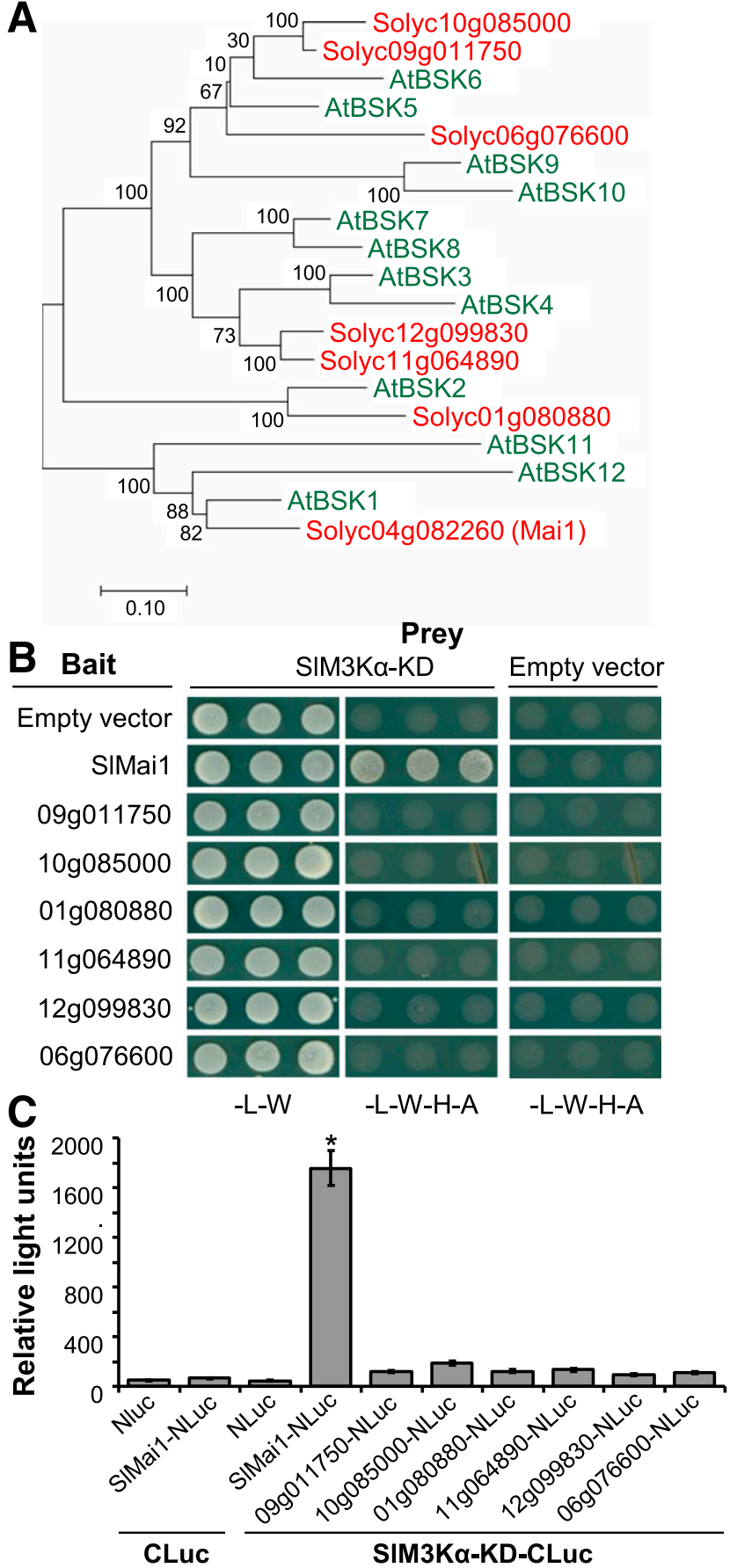

Fig. 2. SIM3K $\alpha$ interacts with SIMai1 but not with other tomato brassinosteroid-signaling kinases (BSKs). A, Phylogenetic analysis of tomato (Solanum lycopersicum [Solyc]) and Arabidopsis thaliana (At) BSK family members based on gene coding sequences. Numbers next to the branches indicate the percentage of trees in which the associated taxa are clustered together, and the tree is drawn to scale with the branch lengths measured in the number of substitutions per site. B, Interaction of tomato BSK proteins with SIM3Ka in a yeast two-hybrid assay, using full-length tomato BSKs with the SIM3K $\alpha$-kinase domain $(\mathrm{KD})$. Yeast were grown in medium lacking leucine and tryptophan $(-\mathrm{L}-\mathrm{W})$ or lacking leucine, tryptophan, histidine, and adenine $(-\mathrm{L}-\mathrm{W}-\mathrm{H}-\mathrm{A})$. Empty vectors served as negative controls. Growth on $-\mathrm{L}-\mathrm{W}-\mathrm{H}-\mathrm{A}$ medium indicates a positive interaction. C, Interaction of tomato BSKs and SIM3K $\alpha$ in a split luciferase complementation assay in Nicotiana benthamiana leaves measured by quantitative luminescence. Protein expression was driven by a $35 \mathrm{~S}$ promoter. Results shown are means \pm standard deviation of three technical replicates. The asterisk indicates a significant difference using a Student's $t$ test $(P<0.01)$. Similar results were observed in three independent experiments. A $\mathrm{N}$-terminal fragment of luciferase (NLuc) was fused to the tomato BSK proteins and C-terminal fragment of luciferase (CLuc) was fused to SIM3Ka-KD. Relative light units are shown. amino acid sequences that are essential for catalysis $(\mathrm{GxGxxG}$, HRD, and DFG motifs), which suggests it may be a pseudokinase (Supplementary Fig. S3) (Bayer et al. 2009; Grütter et al. 2013; Kwon et al. 2019; Sreeramulu et al. 2013). Consistent with this observation and with a recent report about AtBSK1 (Neu et al. 2019), we were unable to detect SIMai1 kinase activity using multiple in-vitro assay conditions, including conditions previously published for AtBSK1 kinase activity (Zhao et al. 2019). TPR motifs consist of a degenerate 34amino acid consensus sequence that forms two antiparallel alpha helices (Blatch and Lässle 1999) and domains often mediate protein-protein interactions (Blatch and Lässle 1999; Kwon et al. 2009; Nyarko et al. 2007). The SIMai1 TPR domain alone was sufficient for interacting with the SIM3K $\alpha$ KD (Fig. 1D). The abolished immunity-related functions in Arabidopsis bsk1-1 mutants were associated with an R443Q substitution in the TPR domain (Shi et al. 2013b), but the comparable substitution (R430Q) in SIMail did not affect its interaction with SlM3K $\alpha$. Immunoblotting showed that all proteins were expressed.

\section{SIM3K $\alpha$ interacts only with SIMai1 among the seven BSK proteins in tomato.}

The tomato genome has seven $B S K$ gene family members, compared with the $12 B S K$ genes present in the Arabidopsis genome (Fig. 2A) (Sreeramulu et al. 2013). The transcript abundance of three of the tomato BSK genes, including SlMail, increases upon activation of the Pto/Prf pathway in tomato (Supplementary Table S1). Although the seven tomato BSK proteins have highly similar TPR sequences (Supplementary Fig. S4), only SIMai1 interacted with SIM3K $\alpha$ in a yeast twohybrid assay (Fig. 2B). The same specificity of the SIM3K $\alpha$ and SIMai1 interaction was observed in a split luciferase complementation assay, in which SIM3K $\alpha$ and the tomato BSK proteins were expressed in $N$. benthamiana leaves using the 35S CaMV (cauliflower mosaic virus) promoter (Fig. 2C). Immunoblotting showed that all proteins were expressed.

SIMai1 interacts with a subset of tomato M3Ks in yeast.

In an initial effort to explore the role of SIMai1, an additional yeast two-hybrid screen of the tomato cDNA library was conducted using SIMai1 as the bait. This screen identified a 14-3-3 protein (TFT3; Solyc04g074510) and, interestingly, another immunity-associated M3K (M3K $\gamma 2$; Solyc02g065110) (Hashimoto et al. 2012). We therefore tested the possible interaction of SIMai1 with additional SIM3Ks. Pairwise yeast two-hybrid assays were performed, using the KDs of six additional SIM3Ks in the MEKK, ZIK, and RAF families as the prey proteins (Supplementary Fig. S5) (Ichimura et al. 2002; Wu et al. 2014). Three of these SIM3Ks interacted strongly with SlMai1. All five SlMai1-interacting SIM3Ks are in the MEKK family. The transcript abundance of two of these interacting SIM3Ks (SIM3K $\alpha$ and Solyc04g079400) is increased during flgII-28-induced pattern recognition receptor-triggered immunity (PTI) as well as during the Pto/Prf-mediated immune response in tomato. A distinguishing amino acid motif is not present in the KDs of the SIM3Ks that interact with SIMai1.

\section{SIMai1 is localized to the cell periphery.}

Myristoylation and palmitoylation sites, as are predicted in the SIMai1 N-terminus, can promote localization of proteins to the plasma membrane (PM). With confocal microscopy, a Mai1-yellow fluorescent protein (YFP) fusion protein was observed to be localized to the plant cell periphery, similar to another RLCK (Solyc10g085990) that has predicted myristoylation and palmitoylation sites (Supplementary Fig. S6). 
Substitutions in the putative S1Mail myristoylation site (G2A) or palmitoylation sites $(\mathrm{C} 3 \mathrm{~S} / \mathrm{C} 4 \mathrm{~S})$ altered its localization, as indicated by YFP signal appearing in the nucleus and the cytoplasm. Immunoblotting showed each protein was expressed similarly in N. benthamiana.

Silencing of Mail homologs in N. benthamiana, using two SlMail fragments, compromises PCD and NTI associated with Pto/Prf and PCD induced by other CC-NLR proteins.

The transcript abundance of many genes with established roles in the Pto/Prf pathway increases upon activation of the Pto/Prf pathway (Supplementary Fig. S7). The transcript abundance of SlMail also increases during this NTI response, and this observation, along with the SIMai1 interaction with SlM3K $\alpha$, suggested that SIMai1 might contribute to plant immunity. To test this hypothesis, two nonoverlapping fragments (SlMail-1 and SlMail-2) from the SlMail gene, each of which is predicted to silence all four of the SIMail homologs present in $N$. benthamiana, were used for tobacco rattle virus (TRV)based VIGS (Fig. 3A; Supplementary Fig. S8). Silencing was confirmed using SlMail-specific primers in semiquantitative reverse transcription (RT)-PCR. SlM $3 K \alpha$ and $S l M K K 2$, whose silencing is known to compromise NTI-associated PCD (del Pozo et al. 2004), and the empty TRV vector were included as controls. Silencing of the Mail homologs in $N$. benthamiana (NbMail), using either SlMail-1 or SlMail-2, or of tomato $M 3 K \alpha(S l M 3 K \alpha)$ and N. tabacum MKK2 (NtMKK2), compromised the PCD normally observed when AvrPto + Pto or AvrPtoB $_{1-387}+$ Pto were coexpressed by agroinfiltration (Fig. $3 \mathrm{~A}$ and $\mathrm{C})$.

$N$. benthamiana recognizes the effector HopQ1, which is present in Pseudomonas syringae pv. tomato DC3000, and deletion of this effector allows DC3000 to cause disease on this species (Wei et al. 2007). Additionally, a N. benthamiana line (R411b) is available that stably expresses both Pto and Prf, thus conferring a strong NTI response to DC3000 through recognition of AvrPto or AvrPtoB (Balmuth and Rathjen 2007). To test the role of Mail in Pto/Prf resistance to $P$. syringae pv. tomato, the SlMail-1 and SIMail-2 VIGS constructs were used to silence the NbMail homologs in $N$. benthamiana $\mathrm{R} 411 \mathrm{~b}$, the plants were inoculated with DC3000 4 hopQ1 or DC3000 $4 h o p Q 1 / \Delta \mathrm{avrPto} / \triangle a v r P t o B$, and the bacterial populations were measured. The DC3000 4 hopQ1 strain (which expresses AvrPto and AvrPtoB) showed significantly increased growth (16-fold more) in NbMail-silenced leaves as compared with unsilenced R411b leaves (Fig. 3B). In contrast, the

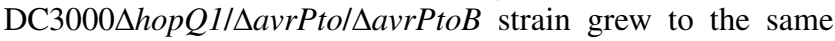
population level in NbMail-silenced and unsilenced leaves. These results support a role for Mail in disease resistance to $P$. syringae pv. tomato mediated by Pto/Prf. A comparable difference in avirulent $P$. syringae pv. tomato growth has been reported for $N$. benthamiana silenced for $\operatorname{Prf}$ (approximately 30-fold) (Lu et al. 2003; Pombo et al. 2014) or NRC2/3 (approximately 10-fold) (Wu et al. 2016) compared with unsilenced plants.

We have reported previously that silencing of $M 3 K a$ or TFT7 in N. benthamiana compromises PCD that is induced by several NLR protein/effector pairs (del Pozo et al. 2004; Oh et al. 2010). The SlMail-1 and SlMail-2 VIGS constructs were therefore used to silence the NbMail homologs in $N$. benthamiana, and the silenced leaves were agroinfiltrated with three CC-NLR gene/effector pairs that activate NTIassociated PCD: Arabidopsis RPP13 and the oomycete effector $A T R 13$, potato Gpa2 and the nematode effector $R B P-1$, and potato $R \times 2$ and the potato virus $\mathrm{X}$ coat protein (Oh and Martin 2011). Each of these pairs caused PCD in the empty TRV vector control plants, and this response was reduced by silencing of

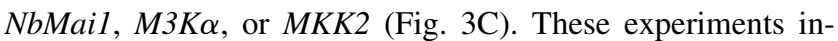
dicate that Mail acts in a pathway shared by several CC-NLR proteins that target diverse pathogens.

\section{Silencing of NbMail homologs does not affect PCD that occurs in $N$. benthamiana leaves upon expression of M3K $\alpha$ or MKK2.}

A MAPK cascade involving M3K $\alpha$ and MKK2 acts downstream of Pto/Prf and plays an important role in activating NTI (del Pozo et al. 2004; Pedley and Martin 2004). Expression of $\mathrm{M} 3 \mathrm{~K} \alpha$ in $N$. benthamiana causes PCD, and this PCD can be compromised by silencing $M K K 2$ (del Pozo et al. 2004). Additionally, expression of the constitutively active form of MKK2 (MKK2 ${ }^{\mathrm{DD}}$ ) also causes PCD in $N$. benthamiana (Oh et al. 2010). To test the position of Mail function in the Pto/Prf

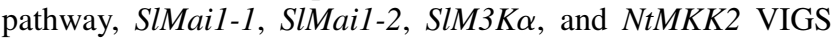
constructs were used for silencing in $N$. benthamiana, and constructs that encode SIM $3 \mathrm{~K} \alpha$ or NtMKK2 ${ }^{\mathrm{DD}}$ were agroinfiltrated into silenced leaves. No decrease in PCD was observed in leaves silenced with SlMail-1 and SlMail-2 and agroinfiltrated with SIM $3 \mathrm{~K} \alpha$ or $\mathrm{NtMKK} 2^{\mathrm{DD}}$, as compared with the control leaves (Fig. 4). As expected, leaves silenced with the $S I M 3 K \alpha$ or NtMKK2 constructs reduced PCD caused by agroinfiltration of SIM3K $\alpha$ and silencing with the NtMKK2 construct compromised PCD caused by NtMKK2 ${ }^{\mathrm{DD}}$ (Fig. 4). These experiments, along with the observations above, indicate that Mai1 acts with M3K $\alpha$ and upstream of MKK2 to regulate NTIassociated PCD.

\section{A synthetic SlMail gene complements PCD impairment} in $N$. benthamiana silenced with SlMail-1 and SlMail-2.

To verify that the phenotypes we observed were not due to silencing of an 'off-target' gene, we developed a synthetic version of SlMail (synSlMail) with a divergent DNA sequence that would make it resistant to silencing, yet encode an identical amino acid sequence (Supplementary Fig. S9). N. benthamiana leaves silenced with the SIMail-1 and SIMail-2 VIGS constructs were agroinfiltrated with SlMail or synSlMail constructs expressing cMyc-tagged SlMail proteins, and the proteins were detected by immunoblotting. As expected, SlMail protein did not accumulate in NbMail-silenced leaves expressing the unaltered SlMail gene but did in leaves expressing synSlMail (Fig. 5A). These observations indicate that synSIMail resists the silencing of the NbMail genes caused by the SlMail-1 and SlMail-2 VIGS constructs. SIMai1 proteins accumulated in unsilenced control plants expressing either SlMail or synSlMail (Fig. 5A).

Changes in nucleotides were introduced into SlMail and synSlMail (Supplementary Table S2) to alter amino acid residues for the myristoylation motif (G2A) and the putative ATP binding site $(\mathrm{K} 91 \mathrm{M})$, to test whether these sites are important for SlMai1 function (a Mai1[C3S/C3S]-myc protein was also tested, but, for unknown reasons, it did not express well). As expected, the variant proteins accumulated in unsilenced control leaves, but only the proteins encoded by synSlMail sequences accumulated in silenced leaves (Fig. 5A).

Next, each of these constructs was agroinfiltrated into leaves of wild-type $N$. benthamiana silenced with the SlMail-1 and SlMail-2 VIGS constructs or unsilenced leaves along with AvrPtoB $_{1-387}$ to induce Pto/Prf-dependent PCD. As expected, in unsilenced control leaves expression of $\mathrm{AvrPtoB}_{1-387}$ with each of the SlMail and synSlMail constructs caused PCD similar to levels observed when AvrPtoB $_{1-387}$ was expressed with a YFP control (Fig. 5B and C). However, in leaves silenced with SlMail-1 and SlMail-2, PCD occurred in areas agroinfiltrated with synSlMail and synSIMail $(K 91 M)$ but not in areas agroinfiltrated with SlMail or a YFP control (Fig. 5B and 
C). Areas agroinfiltrated with synSIMail $(G 2 A)$ developed PCD, but it was reduced compared with synSlMail or synSlMail(K91M) (Fig. 5B and C). In combination with our other localization data, these observations suggest that the glycine-2 myristoylation motif of SIMai1 contributes to SIMai1 function in NTI-associated PCD. Finally, consistent with our inability to detect SIMai1 kinase activity, the ability of the SlMai1(K91M) protein to fully complement Mail silencing indicates that kinase activity of Mail does not play a role in NTI-associated PCD.
AtBSK1 does not restore PCD in $N$. benthamiana plants silenced with SlMail-1 and SlMai1-2.

In light of the relatively high amino-acid sequence identity between SlMail and AtBSK1, we tested whether AtBSK1 could restore immunity-associated PCD in $N$. benthamiana plants silenced with the SlMail-1 and SlMail-2 VIGS constructs. An alignment of the AtBSK1 DNA sequence with the SlMail-1 and SlMail-2 VIGS constructs revealed little overall similarity suggesting AtBSK1 might evade silencing by these constructs (Supplementary Fig. S10). In fact, an immunoblot

\section{A}

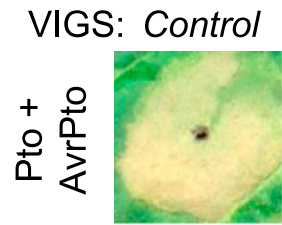

$25 / 25$
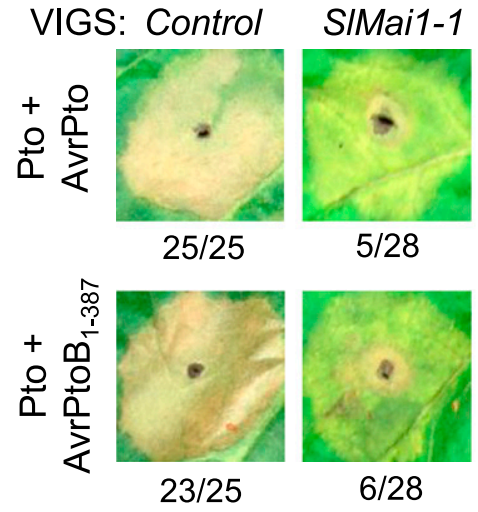

$5 / 28$

C

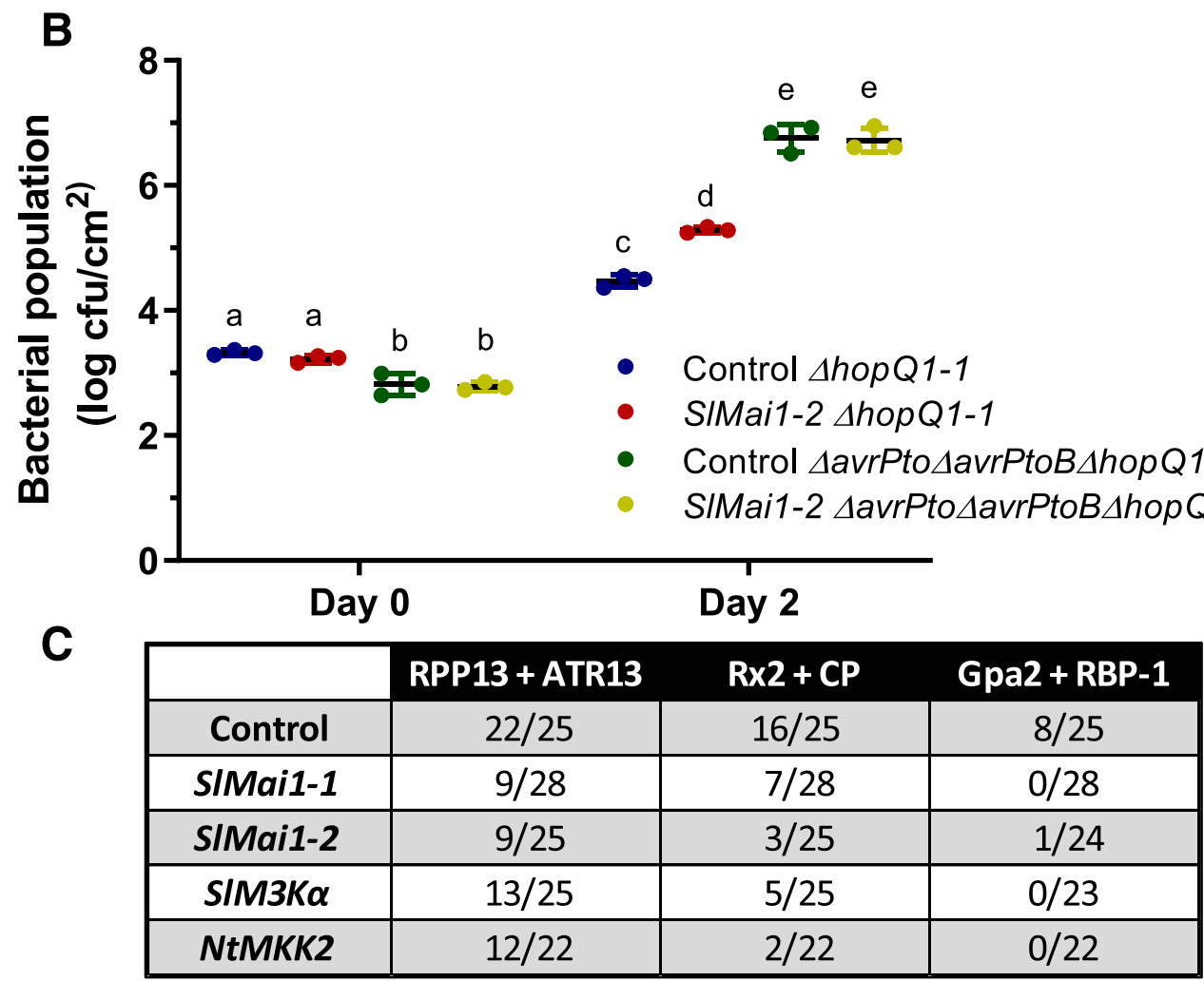

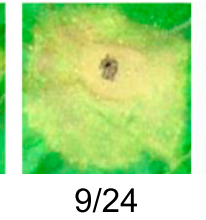

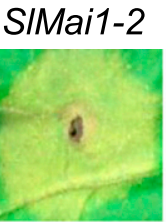

$7 / 24$

$9 / 24$

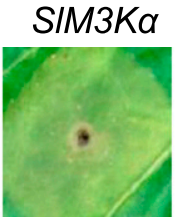

$0 / 25$

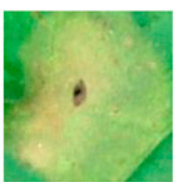

$3 / 25$

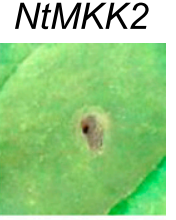

$1 / 22$

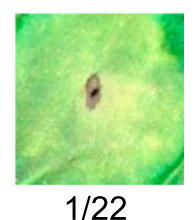

Fig. 3. Mail contributes to programmed cell death (PCD) and disease resistance induced by Pto/Prf and PCD induced by several coiled coil-nucleotide-binding leucine-rich repeat receptor (NLR) proteins. A, The tobacco rattle virus (TRV) virus-induced gene silencing (VIGS) system was used in Nicotiana benthamiana to silence NbMail homologs (using two nonoverlapping constructs, SlMail-1 and SlMail-2), or genes known to reduce PCD (SlM3Ka and SlMKK2), or a TRVonly control. Using the $35 \mathrm{~S} \mathrm{CaMV} \mathrm{(cauliflower} \mathrm{mosaic} \mathrm{virus)} \mathrm{promoter,} \mathrm{PCD} \mathrm{elicitors} \mathrm{were} \mathrm{expressed} \mathrm{by} \mathrm{agroinfiltration} \mathrm{into} \mathrm{leaves} \mathrm{of} \mathrm{silenced} \mathrm{plants.}$ Numbers below photos indicate the number of spots observed with cell death compared with the total number of spots infiltrated from three replicates $(n=22$ to 28). Photographs representative of the most common response in each treatment are shown. B, N. benthamiana plants that stably express Pto and Prf (R411b plants) were silenced with the SlMail-1 and SIMai1-2 VIGS constructs or the TRV control and were infiltrated with Pseudomonas syringae pv. tomato

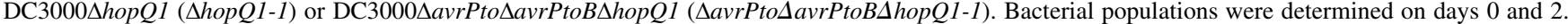
Significance was determined using analysis of variance with a Tukey's post hoc multiple comparisons test, and letters indicate significant differences between treatments $(P<0.001)$. Results shown are the individual values from each plant $(n=3)$ and standard deviation, and means are shown with a horizontal line. Data shown are representative of three biological replicates $(n=9)$. C, VIGS and agroinfiltration of the indicated NLR and Avr cell death elicitors was performed, as in $\mathrm{A}$, with all constructs expressed from the $35 \mathrm{~S} \mathrm{CaMV}$ promoter. Indicated is the number of spots observed with cell death compared with the total number of spots infiltrated from three replicates $(n=22$ to 28$)$. 
comparing SIMai1, synSIMai1, and AtBsk1 expression in Mail-silenced and control plants showed that AtBSK1 protein was expressed in both the control and Mail-silenced plants (Supplementary Fig. S11). We then tested whether AtBSK1 could complement Mail-silenced plants, using the same conditions as described in Figure 5. Unlike synSIMai1, AtBSK1 was unable to restore PCD, suggesting that AtBSK1 and SIMai1 are functionally distinct or, possibly, that AtBSK1 acts with other proteins that are not present or are too divergent in N. benthamiana.

\section{Overexpression of SIMai1 with SIM3Ka}

in $N$. benthamiana accelerates development of PCD

and increases activation of MAPKs, and the SIMai1 myristoylation motif contributes to these responses.

We reported previously that coexpression in $N$. benthamiana leaves of M3K $\alpha$ with its interacting partner TFT7, a 14-3-3 protein, led to increased accumulation of $\mathrm{M} 3 \mathrm{~K} \alpha$ and also accelerated the time at which PCD occurred (Oh et al. 2010). We performed a similar experiment using agroinfiltration of $N$. benthamiana leaves to coexpress SIM3K $\alpha$ or SIM3K $\alpha-\mathrm{KD}$ with SIMai1 (using the synSlMail construct) or YFP as a control. When scored at 48 and $72 \mathrm{~h}$ after SIM3K $\alpha$ or SIM3K $\alpha$ $\mathrm{KD}$ protein induction with estradiol, PCD occurred faster in leaves coexpressing either of the SIM3K $\alpha$ proteins with SIMai1 as compared with the SIM3K $\alpha$ with YFP control infiltrations (Fig. 6A). As expected, no PCD occurred in leaf areas agroinfiltrated with a kinase-inactive $\operatorname{SIM} 3 \mathrm{~K} \alpha-\mathrm{KD}(\mathrm{K} 231 \mathrm{M})$ variant (Fig. 6A). Five days after agroinfiltration, PCD occurred in all agroinfiltrated areas in which it was expected (Supplementary Fig. S12).

The enhanced PCD in this experiment raised the possibility that SIMai1 interaction with SIM3K $\alpha$ increases accumulation of one or both of these proteins or enhances downstream MAPK activity. To test these hypotheses, the SIM3K $\alpha$ and SlMai1 constructs were agroinfiltrated into $N$. benthamiana leaves, and SIM3K $\alpha$ expression was induced with estradiol 2 days after infiltration. At 12, 24, and $30 \mathrm{~h}$ after induction with estradiol, total proteins were extracted and analyzed by immunoblotting. No difference in the abundance of SIM3K $\alpha-\mathrm{KD}$ or SIMai1 protein was observed when they were coexpressed as compared with coexpression with YFP. Employing similar conditions but sampling leaves at $2 \mathrm{~h}$ after estradiol induction of the SIM $3 \mathrm{~K} \alpha$ constructs, we used an antibody that specifically detects phosphorylated (activated) MAPKs and observed that NbMAPK phosphorylation was substantially increased when SlMai1 was coexpressed with SIM3K $\alpha-\mathrm{KD}$; a slight increase in
NbMAPK phosphorylation was observed upon coexpression of SIMai1 with full-length SIM3K $\alpha$ (Fig. 6B). Consistent with the complementation experiments in Figure 5, coexpression of SIMai1(G2A) with SIM3K $\alpha$ did not cause an increase in MAPK phosphorylation, whereas coexpression of SIMai1(K91M) with SIM3K $\alpha$ was just as effective as SIMai1 in enhancing NbMAPK phosphorylation (Fig. 6C). We attempted to test the requirement of the SIMai1 TPR domain for PCD enhancement and activation of M3K $\alpha$, but a SIMai1 protein lacking the TPR domain was poorly expressed in $N$. benthamiana leaves (Supplementary Fig. S13)

\section{DISCUSSION}

We discovered that the tomato Mai1 protein interacts in vivo in both a yeast two-hybrid system and a plant split luciferase complementation assay with tomato $\mathrm{M} 3 \mathrm{~K} \alpha$, a previously described positive regulator of NTI (del Pozo et al. 2004). The SlMai1 TPR domain and the SIM3K $\alpha$ KD are sufficient for mediating their interaction. Although all seven tomato BSK proteins have a TPR domain, SIM $3 \mathrm{~K} \alpha$ interacts only with SlMai1. In contrast, SIMai1 interacts with at least five members of the MEKK family. The predicted SIMai1 fatty acylation sites play a role in its localization to the cell periphery. Experiments in $N$. benthamiana indicated that, like SIM3K $\alpha$ and TFT7, Mail is a positive regulator of PCD induced by several CCNLR proteins. Complementation assays using a synthetic SlMail gene that resists silencing induced by the SlMail-1 and SlMail-2 VIGS constructs confirmed that SIMai1 contributes to immunity-associated PCD and indicated that fatty acylation of SIMai1 but not kinase activity is important for its function. Importantly, we found that Mail contributes to P. syringae pv. tomato resistance conferred by Pto/Prf. Coexpression of SIMai1 with SIM3K $\alpha-K D$ does not affect the accumulation of either protein, although it does enhance phosphorylation of downstream MAPKs. The observed effect was stronger with SIM3K $\alpha-K D$ than full-length M3K $\alpha$, which may be due to the higher expression levels of SIM $3 \mathrm{~K} \alpha-\mathrm{KD}$ compared with SIM3K $\alpha$, as shown previously (del Pozo et al. 2004), and the short expression time frame $(2 \mathrm{~h})$. Together, these observations reveal Mai1 as a new component of the plant immune system, acting between CC-NLR proteins and a MAPK signaling pathway that contributes to PCD and resistance to $P$. syringae pv. tomato.

The first indication that BSK1-like proteins might play a role in NTI came from the discovery that AtBSK1 occurs in a complex containing RPS2, although this observation has not

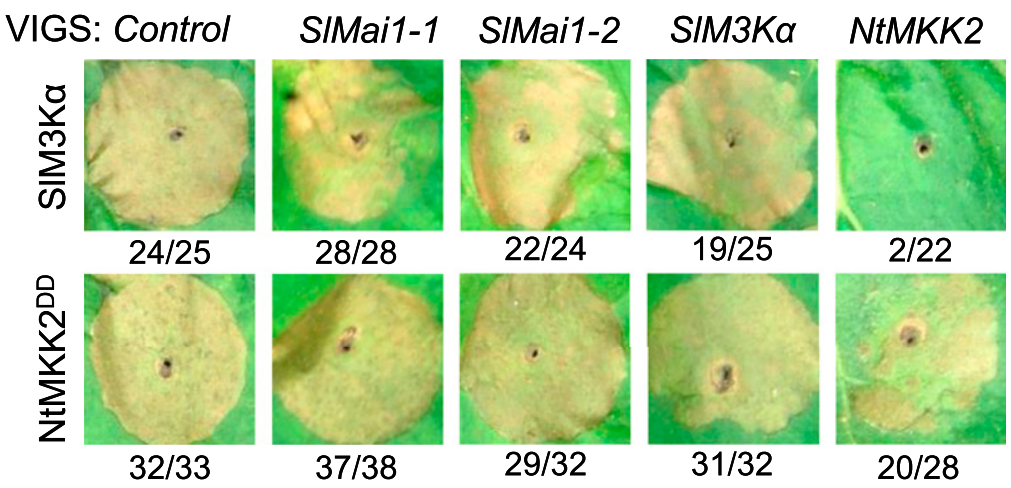

Fig. 4. Mai1 acts upstream of $\mathrm{M} 3 \mathrm{~K} \alpha$ and $\mathrm{MKK} 2$. Representative photographs of the cell death observed 5 days postagroinfiltration of the cell death elicitors $\mathrm{S} 1 \mathrm{M} 3 \mathrm{~K} \alpha$ or constitutive-active NtMKK $2^{\mathrm{DD}}$ into $N$. benthamiana leaves that were silenced using the indicated VIGS constructs. Expression of cell-death elicitors was driven by a $35 \mathrm{~S}$ cauliflower mosaic virus promoter. Indicated are the number of spots observed with cell death compared with the total number of spots infiltrated from three replicates $(n=22$ to 38$)$. Photographs representative of the most common response in each treatment are shown. 
been investigated further (Qi et al. 2011). Subsequent studies in Arabidopsis showed that both bsk1-1 and mapkkk5 mutants were more susceptible to DC3000 strains expressing AvrRpt2 or AvrRphB, which are recognized by the CC-NLRs RPS2 and RPS5, respectively (Shi et al. 2013b; Yan et al. 2018). However, the bsk1-1 and mapkkk5 mutants were similarly more susceptible to virulent DC3000, so the specific connection to NTI was unclear. In $N$. benthamiana, silencing of $N b B S K 1, N b M K K 2$, and $N b S I P K$ blocked brassinosteroid-induced tobacco mosaic virus resistance (Deng et al. 2016). We tested whether AtBSK1 could restore PCD in $N$. benthamiana plants silenced with the SlMail-1 and SlMail-2 VIGS constructs and found that AtBSK1, though expressed well in these plants, could not restore PCD like synSIMai1, suggesting that SIMai1 and AtBSK1 have at least some distinct functions in Arabidopsis and tomato. However, it is also possible that the failure of AtBSK1 to complement Mail-silencing is due to protein incompatibility between Arabidopsis and tomato. Our observations that Mai1 plays a role in PCD associated with several CC-NLR proteins and contributes to $P$. syringae pv. tomato resistance conferred by the Pto/Prf pathway support a role in NTI signaling, and the interaction of Mai1 with M3K $\alpha$ sheds lights on the mechanism for its function in enhancing MAPK activation leading to immunity-associated PCD.

Our finding that the SIMai1 TPR domain is necessary and sufficient for interaction with $\operatorname{SIM} 3 \mathrm{~K} \alpha$ is consistent with the known role of TPRs in mediating protein-protein interactions for diverse biological processes including immune responses (SGT1 [Azevedo et al. 2006]) and hormone signaling (AtTRP1 [Lin et al. 2009]) in plants. The Arabidopsis bsk1-1 mutant allele encodes a protein with an R443Q substitution in its TPR domain, yet this alteration did not affect the interaction of AtBSK1 with FLS2 (Shi et al. 2013b). We made the

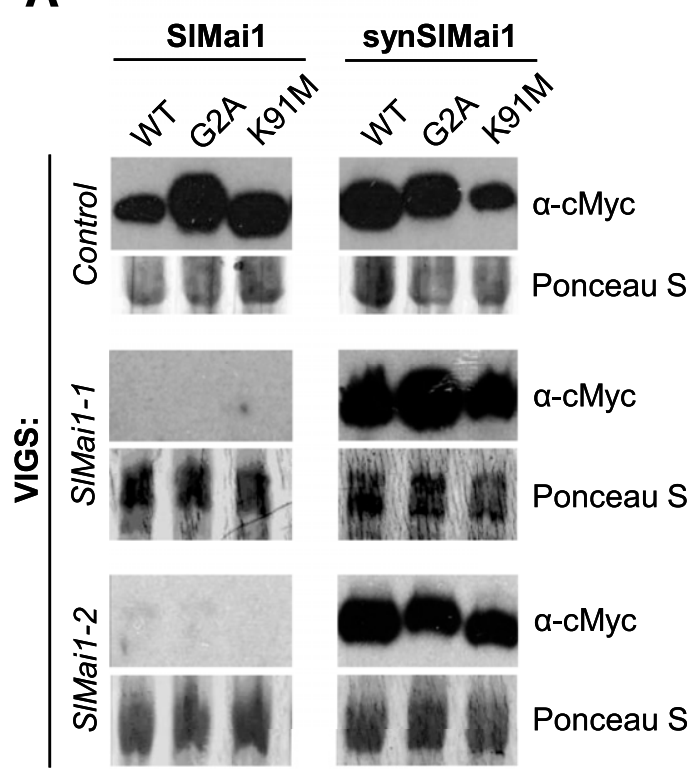

comparable substitution (R430Q) in SIMail and observed no effect on the interaction of SIMai1 with SIM3K $\alpha$ in a yeast twohybrid assay. Further studies are therefore needed to determine whether this residue plays a role in SIMail function. The SIMai1 TPR domain consists of approximately 100 amino acids and contains three TPR motifs. The amino acid sequences of the TPR domains for the seven tomato BSK proteins are remarkably similar, although there are 13 residues unique to SlMai1. Specificity for the interaction of the SIMai1 TPR domain with the KD of $\operatorname{SIM} 3 \mathrm{~K} \alpha$ should facilitate the future identification of the specific TPR amino acids important for binding SIM3K $\alpha$ and may provide insight into how this interaction enhances MAPK activation.

The KDs of five of the nine tested SIM3Ks interacted with SIMai1. While this work focused on the role of SIMai1 in NTI, these multiple interactions might explain how Mai1 (and BSK1 proteins in other species) may contribute to both PTI and NTI responses, and future work will address whether SIMai1 plays a role in PTI. Some of the SIM3Ks that interacted with SIMai1 have been associated with diverse plant immune responses. For example, $\mathrm{M} 3 \mathrm{~K} \alpha$, in addition to contributing to CC-NLR pathways, also plays a role in resistance to Plantago asiatica mosaic virus in $N$. benthamiana (Hashimoto et al. 2012; Komatsu et al. 2010). In addition, the RLCK AtPBL27 interacts with the $\mathrm{KD}$ of AtM3K $\alpha$ and the C-terminal domain of AtMAPKKK5 (M3K $\gamma)$, with the latter interaction providing a molecular link between chitin perception via the CERK1 receptor and downstream MAPK signaling (Yamada et al. 2016). $\mathrm{M} 3 \mathrm{~K} \gamma$ and $\mathrm{M} 3 \mathrm{~K} \alpha$ also act in a M3K $\beta>\mathrm{M} 3 \mathrm{~K} \alpha>\mathrm{M} 3 \mathrm{~K} \gamma$ PCDinducing signaling pathway in $N$. benthamiana (Hashimoto et al. 2012). Since TPR domains can promote self-association (Nyarko et al. 2007), it would be interesting to test if Mai 1 self-association facilitates the interaction of $\mathrm{M} 3 \mathrm{~K} \alpha$ and $\mathrm{M} 3 \mathrm{~K} \gamma$
A B

\section{B}

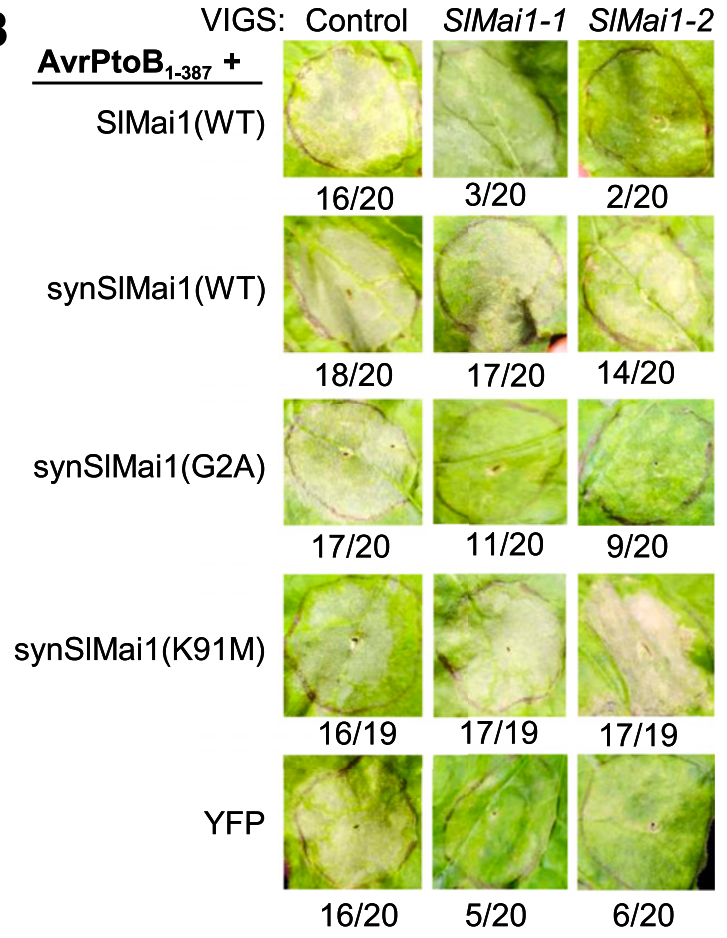

Fig. 5. Synthetic SIMail complements cell death impairment in Nicotiana benthamiana plants silenced with the SlMail-1 and SlMail-2 virus-induced gene silencing (VIGS) constructs. A, Immunoblot analysis showing that proteins accumulate from the expression of constructs encoding synthetic SlMail (synSIMail) but not SlMail in N. benthamiana plants silenced with the VIGS constructs SlMai1-1 or SlMail-2. SIMai1-cMyc fusion proteins were detected using an anti-cMyc antibody. Equal loading was confirmed by Ponceau S stain. B, Cell death induced by AvrPtoB $1-387$ was recovered by SlMai1 proteins expressed from synSlMail or synSlMail(K91M) in wild-type N. benthamiana plants silenced with the VIGS constructs SlMai1-1 or SlMai1-2. All constructs were agroinfiltrated into $N$. benthamiana leaves. Expression was driven with a $35 \mathrm{~S}$ promoter. Photographs are representative of three independent experiments with similar results. Indicated are the number of spots observed with cell death compared with the total number of spots infiltrated from three replicates $(n=19$ or 20). Photographs representative of the most common response in each treatment are shown. 
in this pathway. In Arabidopsis, the M3K YODA regulates several immune responses, and a constitutive-active version of this protein confers broad-spectrum resistance to multiple pathogens, including $P$. syringae pv. tomato (Sopeña-Torres et al. 2018). We did not test the interaction of SIMai1 with the YODA homolog from tomato (Solyc08g081210), although SIMai1 did interact with a related protein, YODA2 (Solyc03g025360). Interestingly, it was recently reported that the AtBSK1 TPR domain interacts with the KD of YODA and plays a role in embryonic patterning in Arabidopsis (Neu et al. 2019). SIMai1

A

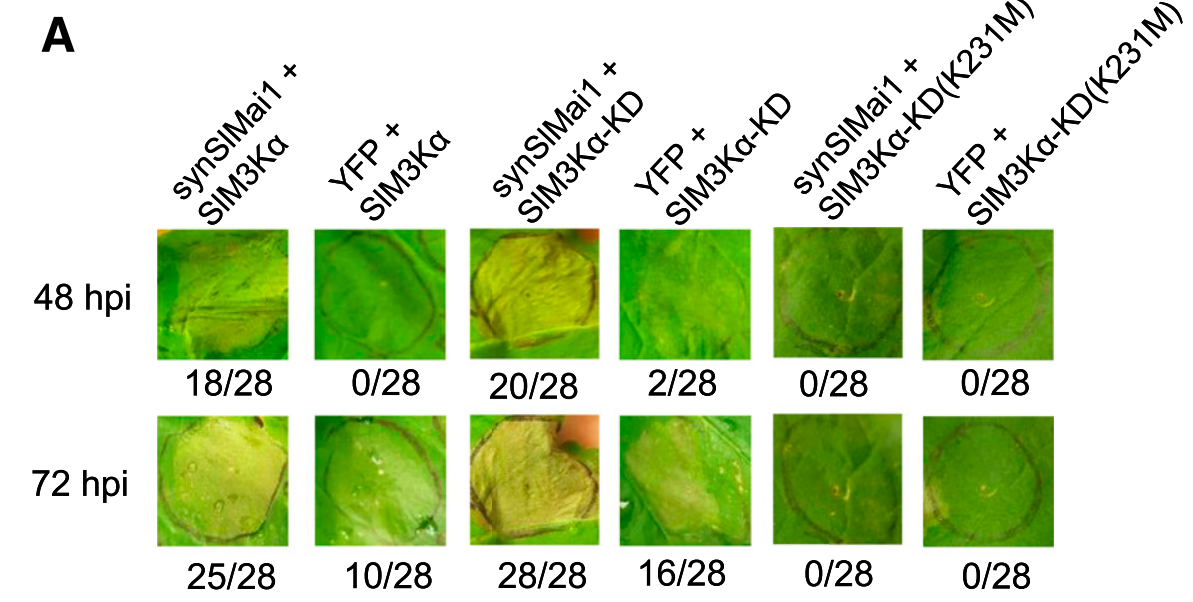

B

SIM3Ka-KD

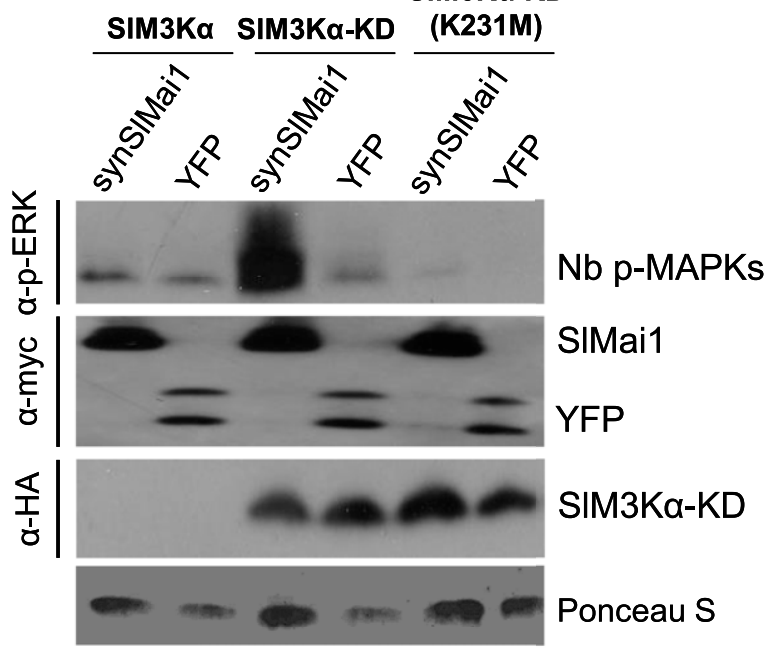

C

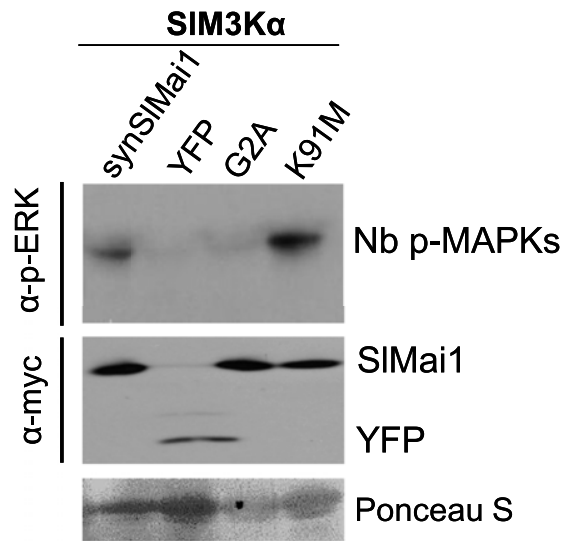

Fig. 6. Overexpression of SIMai1 with SIM3K $\alpha$ in Nicotiana benthamiana accelerates development of programmed cell death and increases activation of mitogen-activated protein kinases (MAPKs), and the SIMail myristoylation motif contributes to these responses. A, Host responses to agroinfiltration of

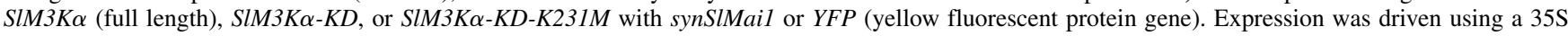

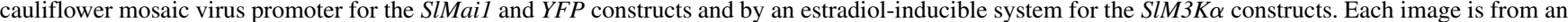
individual infiltration and plant, shown at 48 and $72 \mathrm{~h}$ post-estradiol induction (hpi) of the SIM3K $\alpha$ variants. Indicated are the number of spots that showed cell death compared with the total number of infiltrated spots from three replicates $(n=28)$. Photographs representative of the most common response in each treatment are shown. B, Immunoblot from coexpression of SIM3K $\alpha$ and synSlMail in leaves of $N$. benthamiana. Phosphorylated MAPKs in N. benthamiana ( $\mathrm{Nb}$ p-MAPKs) were detected using an anti-pERK antibody. An anti-cMyc antibody was used to detect the synthetic SiMai1-cMyc constructs, and antihemagglutinin (HA) was used to detect the SIM3K $\alpha$-HA constructs. C, Immunoblot as in B but from coexpression of synSlMail variants (G2A and K91M) with $\operatorname{SlM} 3 K \alpha$. 
did not interact with SIM3Ke which, like SIM3K $\alpha$ and SIMai1, acts downstream of multiple NLRs including Pto/Prf (MelechBonfil and Sessa 2010). M3Ke has been suggested to act in a parallel pathway and not redundantly to $\mathrm{M} 3 \mathrm{~K} \alpha$, and our data suggest it probably uses a different mechanism to activate MAPKs (Melech-Bonfil and Sessa 2010). A distinguishing amino-acid motif is not present in the KDs of the five SIMailinteracting SIM3Ks, as compared with the four that did not interact with SIMai1, but the fact that the five interact with SIMai1 suggests they likely have a common structural feature. In the future, the interaction of SIMai1 with only a subset of the MEKK family of SIM3Ks should facilitate the identification of one or more features in the KD that are involved in the interaction with the SIMai1 TPR domain and may help reveal how this interaction enhances MAPK activation.

The interactions of SIMai1 with multiple tomato M3Ks, including some that do not have known roles in immunity, raise the possibility that SIMai1 (and BSK1-related proteins in other species, as supported by the interaction of BSK1 with YODA in Arabidopsis [Neu et al. 2019]) might have additional functions in other plant processes. In Arabidopsis, AtBSK1 is a substrate of the BRI1 receptor kinase that initiates the brassinosteroid signaling pathway; this pathway has been completely defined and does not involve a M3K (Clouse 2011). Arabidopsis plants with loss-of-function mutations in $B S K l$ and rice plants with reduced expression of BSK1-2 do not have pronounced growth defects, although leaves of the Arabidopsis bsk1-1 mutant are slightly narrower than in wild-type plants and an AtBSK1 genomic clone can complement this phenotype (Shi et al. 2013b; Sreeramulu et al. 2013; Yan et al. 2018). We have observed that $N$. benthamiana plants silenced with the SlMail-1 and SlMail-2 VIGS constructs are slightly smaller than unsilenced control plants and have slightly larger leaves. As part of this work, we generated a CRISPR-induced mutation in SIMail in tomato and found that plants carrying homozygous mail mutations grew more slowly than wild-type plants and showed severe morphological defects with brittle, thin leaves that had distorted shapes (Supplementary Fig. S14). Experiments to test the immunity responses of the mail tomato plants were inconclusive because even mock inoculation caused the leaves to fall off. Interestingly, mail mutant tomato plants that happened to be in a greenhouse sprayed with the insecticide Overture developed severe necrosis and subsequent stunting, as compared with wild-type or Mail/ mail heterozygous plants. We were only able to recover one mail mutant line, and additional characterization of multiple independent mutants will be needed to verify that the mail mutation is responsible for these phenotypes; however, our preliminary observations suggest that SIMail has a role in development or stress responses, or both, in tomato, which is reminiscent of the dual roles of several other proteins in both immunity and development (Huot et al. 2014; Lin et al. 2013).

There are contrasting results in the literature about kinase activity of BSK proteins. We were unable to detect SIMail autophosphorylation or its phosphorylation of the generic kinase substrate myelin basic protein, using multiple in-vitro kinase assay conditions. BSK proteins lack key amino acids that are required for kinase activity and, thus, have been predicted to be pseudokinases with putative roles as scaffold proteins (Bayer et al. 2009; Grütter et al. 2013; Kwon et al. 2019; Sreeramulu et al. 2013). Although AtBSK1 also lacks these motifs, three papers report that AtBSK1 autophosphorylates and can transphosphorylate AtMAPKKK5 and that the ATP binding site was required for its role in resistance to a fungal pathogen and PTI (Shi et al. 2013b; Yan et al. 2018; Zhao et al. 2019). We attempted to reproduce the kinase activity reported for AtBSK1 but were unable to see activity under our kinase assay conditions or those used for AtBSK1 (Zhao et al.
2019). Similarly, a recent report describing the interaction of AtBSK1 with YODA was unable to detect kinase activity for AtBSK1 (Neu et al. 2019). The lack of SlMai1 kinase activity is consistent with other reports of the inability to detect kinase activity for six of the Arabidopsis BSKs (Bayer et al. 2009; Sreeramulu et al. 2013) and with our complementation experiments that showed that disruption of the canonical ATP binding site of SIMai1 did not impact its ability to restore PCD. Further experiments are needed to investigate the contrasting results of SIMai1 and AtBSK1 kinase activity.

Both our microscopy and complementation experiments suggest that SIMail function requires its localization to the cell periphery, at least for its involvement in the Pto/Prf pathway. Fatty acylation and targeting to the PM plays an important role in the function of many immunity-associated proteins (Boyle and Martin 2015; Boyle et al. 2016). AtBSK1 is localized to the $\mathrm{PM}$, and substitutions in its putative myristoylation site (G2A) disrupt this localization and its association with FLS2 (Shi et al. 2013a and b). In the Pto/Prf pathway, both AvrPto and Pto have an $\mathrm{N}$-terminal myristoylation site that is required for their function, and AvrPto is known to localize to the PM (Martin 2012); interestingly, AvrPto has been reported to interact with AtBSK1 (Xiang et al. 2011). It is possible that localization of SlMai1 to the cell periphery promotes its association with certain NLR protein complexes. We observed no interaction of SlMai1 with Pto in a yeast two-hybrid assay, although we were unable to test its possible interaction with Prf in these experiments, since Prf does not express well in yeast. If Mai1 does occur in NLR protein complexes, then it is likely SIMai1 is not localized exclusively to the PM, as AvrPtoB is not PMlocalized and CC-NLRs are known to exist in multiple subcellular compartments (Qi and Innes 2013).

The SIMai1 interaction with SIM3K $\alpha$ and its ability to enhance MAPK signaling provide some initial insight into the mechanism of SIMai1 in the Pto/Prf pathway and open several avenues to further investigate early signaling events acting between NLRs and MAPK signaling. Our current data show that SIMai1 acts directly with SIM3K $\alpha$ but additional experiments are needed to further understand how SIMai1 functions to connect CC-NLR recognition of pathogen effectors to the MAPK signaling cascade (a proposed model is presented in Supplementary Figure S15). However, we demonstrate that Mail is a key player in CC-NLR mediated immunity. AtBSK1 has been found in a complex with RPS2 (Qi et al. 2011) and, as mentioned above, it is possible that SlMail resides in the Pto/Prf complex and perhaps in other CC-NLR complexes. Such an association could stabilize the complexes, facilitate NLR oligomerization, or promote the interaction of NLRs with other proteins, for example Prf with Pto and Fen. SGT1 interacts with Prf (Kud et al. 2013), and the association of the TPR domain in SGT1 and the SIMai1 TPR domain could play a role in SlMail function. The Epk1 kinase is a component of the Pto/Prf pathway that also acts upstream or with M3K $\alpha$ (Pombo et al. 2014). In addition, the NLR proteins NRC2 and NRC3 act in the Pto/Prf signaling pathway, although it is unknown whether they act upstream of MAPK signaling (Wu and Kamoun 2019; Wu et al. 2016, 2017). Investigation of the relationship of Mai1 to Epk1 and NRC2 and NRC3 is an important future goal for understanding the molecular links between host recognition of effectors and MAPK signaling.

\section{MATERIALS AND METHODS}

\section{Yeast two-hybrid assays.}

Protein interaction and $\beta$-galactosidase assays were performed as described previously (Oh et al. 2010). For the yeast two-hybrid screen using full-length Mail as the bait protein in 
pEG202, a prey library generated from Rio Grande-PtoR tomato leaves inoculated with $P$. syringae pv. tomato DC3000, as previously described (Zhou et al. 1995), was used to screen for SIMail interacting proteins. Tomato BSK genes and M3K $\alpha-$ KD were analyzed in the GAL4 system using bait and prey vectors pGBKT7 and pGADT7, respectively. Further details may be found in the Supplementary Methods.

\section{Phylogenetic analyses.}

Sequence alignments were performed using MUSCLE (Edgar 2004) and maximum likelihood trees were generated with MEGA7 (Kumar et al. 2016).

\section{Split luciferase complementation assay.}

Transient protein expression and luciferase assays were performed as described (Teper et al. 2018). Agrobacterium tumefaciens strains carrying NLuc and CLuc constructs were infiltrated into leaves of $N$. benthamiana. Leaves coexpressing different constructs were examined for luciferase (LUC) activity $40 \mathrm{~h}$ after infiltration (before cell death occurred in the case of SIMai1 and $\mathrm{M} 3 \mathrm{~K} \alpha$ ). Quantitative LUC activity was determined by a Veritas Microplate Luminometer (Promega, Madison WI, U.S.A.) and reported as relative light units.

\section{Virus-induced gene silencing (VIGS).}

The TRV vector derivatives were transformed into A. tumefaciens GV2260 and prepared for infection as previously described (Chakravarthy et al. 2010). Cell death, transient expression, and bacterial growth assays were performed five-to-six weeks after agroinfiltration with TRV. To confirm silencing of the NbMail orthologs using the SlMail-1 and SlMail-2 VIGS constructs, semiquantitative RT-PCR was performed as in (del Pozo et al. 2004) using $N b E F 1 \alpha$-specific primers as a loading control.

\section{Cell death assays.}

The various cell death elicitors were agroinfiltrated in $N$. benthamiana TRV-VIGS plants as previously described (Oh et al. 2010). Expression of the cell death elicitors was induced with estradiol at 48 or $62 \mathrm{~h}$ post infiltration (Figs. 3, 4, 5, and 6). All cell death data were visually collected and scored blindly. Further details may be found in Supplementary Text.

\section{In vitro protein kinase assays.}

MBP- or GST- tagged protein plasmids were transformed into BL21 cells for in vitro protein expression. Details on the expression and purification of the proteins can be found in the Supplementary Methods. Kinase assays were performed for $30 \mathrm{~min}$ at room temperature in $20 \mu \mathrm{L}$ of kinase reaction buffer (50mM HEPES, $\mathrm{pH} 7.5,10 \mathrm{mM} \mathrm{MgCl}_{2}$ and/or $10 \mathrm{mM} \mathrm{MnCl}_{2}$ and/or $10 \mathrm{mM} \mathrm{CaCl} 2$, and $3 \mu \mathrm{g}$ myelin basic protein) containing $2 \mu \mathrm{Ci}\left[\gamma^{32} \mathrm{P}\right]$, or were performed exactly as described in (Zhao et al. 2019), using $5 \mu \mathrm{g}$ of each of the proteins. Reactions were stopped by adding SDS-PAGE sample buffer. Samples electrophoresed on a $10 \%$ SDS-PAGE gel. Gels were dried using a gel dryer and radiolabeled proteins were visualized on autoradiography film or a phosphoimaging system.

\section{Generation of synthetic Mai1 and sequence variants.}

The synthetic SlMail sequence was initially designed using the Integrated DNA Technologies (IDT) Codon Optimization Tool, and individual nucleotides were then changed such that no fragment longer than 11 nucleotides was identical to the SlMail sequence. Synthetic SlMail variants were generated by PCR amplification using oligonucleotides encoding the amino acid changes. Further cloning details can be found in the Supplementary Methods.

\section{Immunoblots of transiently expressed proteins in N. benthamiana.}

Total protein was extracted from A. tumefaciens-infiltrated leaves as previously described (Hind et al. 2016), and $10 \mu \mathrm{g}$ was loaded on SDS-PAGE before transferring to PVDF membrane (Merck Millipore). SlMai1-myc or synSIMai1myc proteins were detected using anti-Myc antibodies (GenScript) and chemilumiscent ECL Plus substrate (Thermo Fisher Scientific). Phosphorylated (activated) MAPK proteins were detected using antiphospho-p44/42 MAPK T202/Y204 (pERK) antibodies (Cell Signaling). SIM3K $\alpha$ proteins were detected using anti-hemagglutinin antibodies (Krackeler). Membranes were stained with Ponceau S (Sigma Aldrich) to verify equal loading.

\section{Bacterial population assays.}

Six-week-old SlMail-2-silenced or control-silenced plants were infiltrated with $P$. syringae pv. tomato strains DC3000 4 hopQ1,

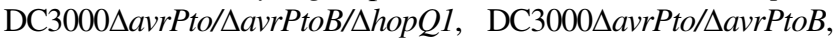
or DC3000 $\Delta a v r P t o / \Delta a v r P t o B / \Delta h o p Q 1$, using a 1:10,000 dilution at an optical density at $600 \mathrm{~nm}$ of 0.5 (approximately $1 \times 10^{4}$ $\mathrm{CFU} / \mathrm{ml}$ ). Three leaf discs (each $0.33 \mathrm{~cm}^{2}$ ) from each replicate plant were taken $2 \mathrm{~h}$ after infiltration (day 0 ) and 2 or 3 days later and were homogenized in $10 \mathrm{mM} \mathrm{MgCl}$ to determine the bacterial populations via serial dilution plating. A two-tailed Student's $t$ test was used to calculate the $P$ values.

\section{ACKNOWLEDGMENTS}

We thank F. Rinaldi for supporting research and helpful comments on the manuscript, T. Jacobs, R.-A. Langan, N. Zhang, H. Roberts, and F. Giska for performing supporting experiments, B. Bell and J. Miller for greenhouse assistance, A. Liu and K. Chen for experimental assistance, and J. Rathjen for R411b seeds.

\section{AUTHOR RECOMMENDED RESOURCES}

Boyce Thompson Institute Nicotiana benthamiana genomics resources: https://btiscience.org/our-research/research-facilities/researchresources/nicotiana-benthamiana

IDT Codon Optimization Tool: https://www.idtdna.com/pages/tools

Plant Genome Editing Database (PGED): http://plantcrispr.org/cgi-bin/crispr/index.cgi

Sol Genomics Network (SGN) database: http://solgenomics.net

Tomato Functional Genomics Digital expression (RNA-seq) experiment list database: http://ted.bti.cornell.edu/cgi-bin/TFGD/digital/home.cgi

SGN VIGS tool: http://vigs.solgenomics.net

\section{LITERATURE CITED}

Aarts, N., Metz, M., Holub, E., Staskawicz, B. J., Daniels, M. J., and Parker, J. E. 1998. Different requirements for EDS1 and NDR1 by disease resistance genes define at least two $R$ gene-mediated signaling pathways in Arabidopsis. Proc. Natl. Acad. Sci. U.S.A. 95:10306-10311.

Abramovitch, R. B., and Martin, G. B. 2005. AvrPtoB: A bacterial type III effector that both elicits and suppresses programmed cell death associated with plant immunity. FEMS Microbiol. Lett. 245:1-8.

Agrawal, G. K., Iwahashi, H., and Rakwal, R. 2003. Rice MAPKs. Biochem. Biophys. Res. Commun. 302:171-180.

Azevedo, C., Betsuyaku, S., Peart, J., Takahashi, A., Noël, L., Sadanandom, A., Casais, C., Parker, J., and Shirasu, K. 2006. Role of SGT1 in resistance protein accumulation in plant immunity. EMBO J. 25: 2007-2016.

Balmuth, A., and Rathjen, J. P. 2007. Genetic and molecular requirements for function of the Pto/Prf effector recognition complex in tomato and Nicotiana benthamiana. Plant J. 51:978-990.

Bayer, M., Nawy, T., Giglione, C., Galli, M., Meinnel, T., and Lukowitz, W. 2009. Paternal control of embryonic patterning in Arabidopsis thaliana. Science 323:1485-1488. 
Blatch, G. L., and Lässle, M. 1999. The tetratricopeptide repeat: A structural motif mediating protein-protein interactions. BioEssays 21: 932-939.

Boyle, P. C., and Martin, G. B. 2015. Greasy tactics in the plant-pathogen molecular arms race. J. Exp. Bot. 66:1607-1616.

Boyle, P. C., Schwizer, S., Hind, S. R., Kraus, C. M., De la Torre Diaz, S., He, B., and Martin, G. B. 2016. Detecting N-myristoylation and Sacylation of host and pathogen proteins in plants using click chemistry. Plant Methods 12:38

Brendolise, C., Martinez-Sanchez, M., Morel, A., Chen, R., Dinis, R., Deroles, S., Peeters, N., Rikkerink, E. H. A., and Montefiori, M. 2018. NRG1-mediated recognition of HopQ1 reveals a link between PAMP and effector-triggered Immunity. bioRxiv doi.org/10.1101/293050. Published online.

Castel, B., Ngou, P. M., Cevik, V., Redkar, A., Kim, D. S., Yang, Y., Ding, P., and Jones, J. D. G. 2019. Diverse NLR immune receptors activate defence via the RPW8-NLR NRG1. New Phytol. 222:966-980.

Chakravarthy, S., Velásquez, A. C., Ekengren, S. K., Collmer, A., and Martin, G. B. 2010. Identification of Nicotiana benthamiana genes involved in pathogen-associated molecular pattern-triggered immunity. Mol. Plant-Microbe Interact. 23:715-726.

Chandra, S., Martin, G. B., and Low, P. S. 1996. The Pto kinase mediates a signaling pathway leading to the oxidative burst in tomato. Proc. Natl. Acad. Sci. U.S.A. 93:13393-13397.

Clouse, S. D. 2011. Brassinosteroid signal transduction: From receptor kinase activation to transcriptional networks regulating plant development. Plant Cell 23:1219-1230.

Colcombet, J., and Hirt, H. 2008. Arabidopsis MAPKs: A complex signalling network involved in multiple biological processes. Biochem. J. 413:217-226.

Coll, N. S., Epple, P., and Dangl, J. L. 2011. Programmed cell death in the plant immune system. Cell Death Differ. 18:1247-1256.

del Pozo, O., Pedley, K. F., and Martin, G. B. 2004. MAPKKK $\alpha$ is a positive regulator of cell death associated with both plant immunity and disease. EMBO J. 23:3072-3082.

Deng, X. G., Zhu, T., Peng, X. J., Xi, D. H., Guo, H., Yin, Y., Zhang, D. W., and Lin, H. H. 2016. Role of brassinosteroid signaling in modulating Tobacco mosaic virus resistance in Nicotiana benthamiana. Sci. Rep. 6: 20579.

Dong, O. X., Tong, M., Bonardi, V., El Kasmi, F., Woloshen, V., Wünsch, L. K., Dangl, J. L., and Li, X. 2016. TNL-mediated immunity in Arabidopsis requires complex regulation of the redundant ADR1 gene family. New Phytol. 210:960-973.

Du, X., Miao, M., Ma, X., Liu, Y., Kuhl, J. C., Martin, G. B., and Xiao, F. 2012. Plant programmed cell death caused by an autoactive form of Prf is suppressed by co-expression of the Prf LRR domain. Mol. Plant 5: 1058-1067.

Edgar, R. C. 2004. MUSCLE: Multiple sequence alignment with high accuracy and high throughput. Nucleic Acids Res. 32:1792-1797.

Ekengren, S. K., Liu, Y., Schiff, M., Dinesh-Kumar, S. P., and Martin, G. B. 2003. Two MAPK cascades, NPR1, and TGA transcription factors play a role in Pto-mediated disease resistance in tomato. Plant J. 36:905-917.

Grütter, C., Sreeramulu, S., Sessa, G., and Rauh, D. 2013. Structural characterization of the RLCK family member BSK8: A pseudokinase with an unprecedented architecture. J. Mol. Biol. 425:4455-4467.

Gutierrez, J. R., Balmuth, A. L., Ntoukakis, V., Mucyn, T. S., GimenezIbanez, S., Jones, A. M., and Rathjen, J. P. 2010. Prf immune complexes of tomato are oligomeric and contain multiple Pto-like kinases that diversify effector recognition. Plant J. 61:507-518.

Hashimoto, M., Komatsu, K., Maejima, K., Okano, Y., Shiraishi, T., Ishikawa, K., Takinami, Y., Yamaji, Y., and Namba, S. 2012. Identification of three MAPKKKs forming a linear signaling pathway leading to programmed cell death in Nicotiana benthamiana. BMC Plant Biol. 12:103.

Hind, S. R., Strickler, S. R., Boyle, P. C., Dunham, D. M., Bao, Z., O’Doherty, I. M., Baccile, J. A., Hoki, J. S., Viox, E. G., Clarke, C. R., Vinatzer, B. A., Schroeder, F. C., and Martin, G. B. 2016. Tomato receptor FLAGELLIN-SENSING 3 binds flgII-28 and activates the plant immune system. Nat. Plants 2:16128.

Huot, B., Yao, J., Montgomery, B. L., and He, S. Y. 2014. Growth-defense tradeoffs in plants: A balancing act to optimize fitness. Mol. Plant 7: 1267-1287.

Ichimura, K., Tena, G., Sheen, J., Henry, Y., Champion, A., Kreis, M., Zhang, S., Hirt, H., Wilson, C., Heberle-Bors, E., Ellis, B. E., Morris, P. C., Innes, R. W., Ecker, J. R., Scheel, D., Klessig, D. F., Machida, Y., Mundy, J., Ohashi, Y., and Walker, J. C. 2002. Mitogen-activated protein kinase cascades in plants: A new nomenclature. Trends Plant Sci. 7: 301-308.
Jia, Y., Loh, Y. T., Zhou, J., and Martin, G. B. 1997. Alleles of Pto and Fen occur in bacterial speck-susceptible and fenthion-insensitive tomato cultivars and encode active protein kinases. Plant Cell 9:61-73.

Jia, Y., and Martin, G. B. 1999. Rapid transcript accumulation of pathogenesis-related genes during an incompatible interaction in bacterial speck disease-resistant tomato plants. Plant Mol. Biol. 40: 455-465.

Jones, J. D., Vance, R. E., and Dangl, J. L. 2016. Intracellular innate immune surveillance devices in plants and animals. Science 354: aaf6395.

Komatsu, K., Hashimoto, M., Ozeki, J., Yamaji, Y., Maejima, K., Senshu, H., Himeno, M., Okano, Y., Kagiwada, S., and Namba, S. 2010. Viralinduced systemic necrosis in plants involves both programmed cell death and the inhibition of viral multiplication, which are regulated by independent pathways. Mol. Plant-Microbe Interact. 23:283-293.

Kud, J., Zhao, Z., Du, X., Liu, Y., Zhao, Y., and Xiao, F. 2013. SGT1 interacts with the $\operatorname{Prf}$ resistance protein and is required for $\operatorname{Prf}$ accumulation and Prf-mediated defense signaling. Biochem. Biophys. Res. Commun. 431:501-505.

Kumar, S., Stecher, G., and Tamura, K. 2016. MEGA7: Molecular evolutionary genetics analysis version 7.0 for bigger datasets. Mol Biol. Evol. 33:1870-1874.

Kwon, A., Scott, S., Taujale, R., Yeung, W., Kochut, K. J., Eyers, P. A., and Kannan, N. 2019. Tracing the origin and evolution of pseudokinases across the tree of life. Sci. Signal. 12:eaav3810.

Kwon, S. I., Kim, S. H., Bhattacharjee, S., Noh, J. J., and Gassmann, W. 2009. SRFR1, a suppressor of effector-triggered immunity, encodes a conserved tetratricopeptide repeat protein with similarity to transcriptional repressors. Plant J. 57:109-119.

Lin, W., Ma, X., Shan, L., and He, P. 2013. Big roles of small kinases: The complex functions of receptor-like cytoplasmic kinases in plant immunity and development. J. Integr. Plant Biol. 55:1188-1197.

Lin, Z., Ho, C. W., and Grierson, D. 2009. AtTRP1 encodes a novel TPR protein that interacts with the ethylene receptor ERS1 and modulates development in Arabidopsis. J. Exp. Bot. 60:3697-3714.

Lu, R., Malcuit, I., Moffett, P., Ruiz, M. T., Peart, J., Wu, A. J., Rathjen, J. P., Bendahmane, A., Day, L., and Baulcombe, D. C. 2003. High throughput virus-induced gene silencing implicates heat shock protein 90 in plant disease resistance. EMBO J. 22:5690-5699.

Maekawa, T., Kufer, T. A., and Schulze-Lefert, P. 2011. NLR functions in plant and animal immune systems: So far and yet so close. Nat. Immunol. 12:817-826.

Martin, G. B. 2012. Suppression and activation of the plant immune system by Pseudomonas syringae effectors AvrPto and AvrPtoB. Pages 123-154 in: Effectors in Plant-Microbe Interactions. Martin, F., and Kamoun, S., eds. Wiley-Blackwell, Ames, IA, U.S.A

Mathieu, J., Schwizer, S., and Martin, G. B. 2014. Pto kinase binds two domains of AvrPtoB and its proximity to the effector E3 ligase determines if it evades degradation and activates plant immunity. PLoS Pathog. 10:e1004227.

Melech-Bonfil, S., and Sessa, G. 2010. Tomato MAPKKKE is a positive regulator of cell-death signaling networks associated with plant immunity. Plant J. 64:379-391.

Meng, X., and Zhang, S. 2013. MAPK cascades in plant disease resistance signaling. Annu. Rev. Phytopathol. 51:245-266.

Mucyn, T. S., Clemente, A., Andriotis, V. M., Balmuth, A. L., Oldroyd, G. E., Staskawicz, B. J., and Rathjen, J. P. 2006. The tomato NBARCLRR protein Prf interacts with Pto kinase in vivo to regulate specific plant immunity. Plant Cell 18:2792-2806.

Mucyn, T. S., Wu, A. J., Balmuth, A. L., Arasteh, J. M., and Rathjen, J. P. 2009. Regulation of tomato Prf by Pto-like protein kinases. Mol. PlantMicrobe Interact. 22:391-401.

Neu, A., Eilbert, E., Asseck, L. Y., Slane, D., Henschen, A., Wang, K., Bürgel, P., Hildebrandt, M., Musielak, T. J., Kolb, M., Lukowitz, W. Grefen, C., and Bayer, M. 2019. Constitutive signaling activity of a receptor-associated protein links fertilization with embryonic patterning in Arabidopsis thaliana. Proc. Natl. Acad. Sci. U.S.A. 116 5795-5804.

Ntoukakis, V., Saur, I. M., Conlan, B., and Rathjen, J. P. 2014. The changing of the guard: The Pto/Prf receptor complex of tomato and pathogen recognition. Curr. Opin. Plant Biol. 20:69-74.

Nyarko, A., Mosbahi, K., Rowe, A. J., Leech, A., Boter, M., Shirasu, K., and Kleanthous, C. 2007. TPR-mediated self-association of plant SGT1. Biochemistry 46:11331-11341.

Oh, C. S., and Martin, G. B. 2011. Tomato 14-3-3 protein TFT7 interacts with a MAP kinase kinase to regulate immunity-associated programmed cell death mediated by diverse disease resistance proteins. J. Biol. Chem. 286:14129-14136. 
Oh, C. S., Pedley, K. F., and Martin, G. B. 2010. Tomato 14-3-3 protein 7 positively regulates immunity-associated programmed cell death by enhancing protein abundance and signaling ability of MAPKKK $\alpha$. Plant Cell 22:260-272.

Pedley, K. F., and Martin, G. B. 2003. Molecular basis of Pto-mediated resistance to bacterial speck disease in tomato. Annu. Rev. Phytopathol. 41:215-243.

Pedley, K. F., and Martin, G. B. 2004. Identification of MAPKs and their possible MAPK kinase activators involved in the Pto-mediated defense response of tomato. J. Biol. Chem. 279:49229-49235.

Pedley, K. F., and Martin, G. B. 2005. Role of mitogen-activated protein kinases in plant immunity. Curr. Opin. Plant Biol. 8:541-547.

Pombo, M. A., Zheng, Y., Fernandez-Pozo, N., Dunham, D. M., Fei, Z., and Martin, G. B. 2014. Transcriptomic analysis reveals tomato genes whose expression is induced specifically during effector-triggered immunity and identifies the Epk1 protein kinase which is required for the host response to three bacterial effector proteins. Genome Biol. 15:492.

Qi, D., and Innes, R. W. 2013. Recent advances in plant NLR structure, function, localization, and signaling. Front. Immunol. 4:348.

Qi, T., Seong, K., Thomazella, D. P. T., Kim, J. R., Pham, J., Seo, E., Cho, M. J., Schultink, A., and Staskawicz, B. J. 2018. NRG1 functions downstream of EDS1 to regulate TIR-NLR-mediated plant immunity in Nicotiana benthamiana. Proc. Natl. Acad. Sci. U.S.A. 115: E10979-E10987.

Qi, Y., Tsuda, K., Glazebrook, J., and Katagiri, F. 2011. Physical association of pattern-triggered immunity (PTI) and effector-triggered immunity (ETI) immune receptors in Arabidopsis. Mol. Plant Pathol. 12: 702-708.

Rasmussen, M. W., Roux, M., Petersen, M., and Mundy, J. 2012. MAP kinase cascades in Arabidopsis innate immunity. Front. Plant Sci. 3:169.

Shi, H., Shen, Q., Qi, Y., Yan, H., Nie, H., Chen, Y., Zhao, T., Katagiri, F., and Tang, D. 2013b. BR-SIGNALING KINASE1 physically associates with FLAGELLIN SENSING2 and regulates plant innate immunity in Arabidopsis. Plant Cell 25:1143-1157.

Shi, H., Yan, H., Li, J., and Tang, D. 2013a. BSK1, a receptor-like cytoplasmic kinase, involved in both BR signaling and innate immunity in Arabidopsis. Plant Signal. Behav. 8:e24996.

Sopeña-Torres, S., Jordá, L., Sánchez-Rodríguez, C., Miedes, E., Escudero, V., Swami, S., López, G., Piślewska-Bednarek, M., Lassowskat, I., Lee, J., Gu, Y., Haigis, S., Alexander, D., Pattathil, S., Muñoz-Barrios, A., Bednarek, P., Somerville, S., Schulze-Lefert, P., Hahn, M. G., Scheel, D., and Molina, A. 2018. YODA MAP3K kinase regulates plant immune responses conferring broad-spectrum disease resistance. New Phytol. 218:661-680.

Sreeramulu, S., Mostizky, Y., Sunitha, S., Shani, E., Nahum, H., Salomon, D., Hayun, L. B., Gruetter, C., Rauh, D., Ori, N., and Sessa, G. 2013. BSKs are partially redundant positive regulators of brassinosteroid signaling in Arabidopsis. Plant J. 74:905-919.

Takken, F. L., and Goverse, A. 2012. How to build a pathogen detector: Structural basis of NB-LRR function. Curr. Opin. Plant Biol. 15: 375-384.

Tang, W., Kim, T. W., Oses-Prieto, J. A., Sun, Y., Deng, Z., Zhu, S., Wang, R., Burlingame, A. L., and Wang, Z. Y. 2008. BSKs mediate signal transduction from the receptor kinase BRI1 in Arabidopsis. Science 321: 557-560.

Tena, G., Asai, T., Chiu, W. L., and Sheen, J. 2001. Plant mitogen-activated protein kinase signaling cascades. Curr. Opin. Plant Biol. 4:392-400.

Teper, D., Girija, A. M., Bosis, E., Popov, G., Savidor, A., and Sessa, G. 2018. The Xanthomonas euvesicatoria type III effector XopAU is an active protein kinase that manipulates plant MAP kinase signaling. PLoS Pathog. 14:e1006880.

Tsuda, K., Sato, M., Stoddard, T., Glazebrook, J., and Katagiri, F. 2009. Network properties of robust immunity in plants. PLoS Genet. 5: e1000772. van der Hoorn, R. A., and Kamoun, S. 2008. From guard to decoy: A new model for perception of plant pathogen effectors. Plant Cell 20: 2009-2017.

Wang, J., Hu, M., Wang, J., Qi, J., Han, Z., Wang, G., Qi, Y., Wang, H. W., Zhou, J. M., and Chai, J. 2019a. Reconstitution and structure of a plant NLR resistosome conferring immunity. Science 364:eaav5870.

Wang, J., Shi, H., Zhou, L., Peng, C., Liu, D., Zhou, X., Wu, W., Yin, J., Qin, H., Ma, W., He, M., Li, W., Wang, J., Li, S., and Chen, X. 2017. OsBSK1-2, an ortholog of AtBSK1, is involved in rice immunity. Front. Plant Sci. 8:908.

Wang, J., Wang, J., Hu, M., Wu, S., Qi, J., Wang, G., Han, Z., Qi, Y., Gao, N., Wang, H. W., Zhou, J. M., and Chai, J. 2019b. Ligand-triggered allosteric ADP release primes a plant NLR complex. Science 364: eaav5868.

Wei, C.-F., Kvitko, B. H., Shimizu, R., Crabill, E., Alfano, J. R., Lin, N.-C., Martin, G. B., Huang, H.-C., and Collmer, A. 2007. A Pseudomonas syringae pv. tomato DC3000 mutant lacking the type III effector HopQ11 is able to cause disease in the model plant Nicotiana benthamiana. Plant J. 51:32-46.

Wu, C.-H., Abd-El-Haliem, A., Bozkurt, T. O., Belhaj, K., Terauchi, R., Vossen, J. H., and Kamoun, S. 2017. NLR network mediates immunity to diverse plant pathogens. Proc. Natl. Acad. Sci. U.S.A. 114:8113-8118.

Wu, C.-H., Belhaj, K., Bozkurt, T. O., Birk, M. S., and Kamoun, S. 2016. Helper NLR proteins NRC2a/b and NRC3 but not NRC1 are required for Pto-mediated cell death and resistance in Nicotiana benthamiana. New Phytol. 209:1344-1352.

Wu, C.-H., and Kamoun, S. 2019. Tomato Prf requires NLR helpers NRC2 and NRC3 to confer resistance against the bacterial speck pathogen Pseudomonas syringae pv. tomato. bioRxiv doi.org/10.1101/595744. Published online.

Wu, C.-H., Krasileva, K. V., Banfield, M. J., Terauchi, R., and Kamoun, S. 2015. The "sensor domains" of plant NLR proteins: More than decoys? Front. Plant Sci. 6:134.

Wu, J., Wang, J., Pan, C., Guan, X., Wang, Y., Liu, S., He, Y., Chen, J., Chen, L., and Lu, G. 2014. Genome-wide identification of MAPKK and MAPKKK gene families in tomato and transcriptional profiling analysis during development and stress response. PLoS One 9:e103032.

Wu, Z., Li, M., Dong, O. X., Xia, S., Liang, W., Bao, Y., Wasteneys, G., and Li, X. 2019. Differential regulation of TNL-mediated immune signaling by redundant helper CNLs. New Phytol. 222:938-953.

Xiang, T., Zong, N., Zhang, J., Chen, J., Chen, M., and Zhou, J. M. 2011. BAK1 is not a target of the Pseudomonas syringae effector AvrPto. Mol. Plant-Microbe Interact. 24:100-107.

Xiao, F., He, P., Abramovitch, R. B., Dawson, J. E., Nicholson, L. K., Sheen, J., and Martin, G. B. 2007. The N-terminal region of Pseudomonas type III effector AvrPtoB elicits Pto-dependent immunity and has two distinct virulence determinants. Plant J. 52:595-614.

Yamada, K., Yamaguchi, K., Shirakawa, T., Nakagami, H., Mine, A., Ishikawa, K., Fujiwara, M., Narusaka, M., Narusaka, Y., Ichimura, K., Kobayashi, Y., Matsui, H., Nomura, Y., Nomoto, M., Tada, Y., Fukao, Y., Fukamizo, T., Tsuda, K., Shirasu, K., Shibuya, N., and Kawasaki, T. 2016. The Arabidopsis CERK1-associated kinase PBL27 connects chitin perception to MAPK activation. EMBO J. 35:2468-2483.

Yan, H., Zhao, Y., Shi, H., Li, J., Wang, Y., and Tang, D. 2018. BRASSINOSTEROID-SIGNALING KINASE1 phosphorylates MAPKKK5 to regulate immunity in Arabidopsis. Plant Physiol. 176:2991-3002.

Zhang, X., Dodds, P. N., and Bernoux, M. 2017. What do we know about NODlike receptors in plant immunity? Annu. Rev. Phytopathol. 55:205-229.

Zhao, Y., Wu, G., Shi, H., and Tang, D. 2019. RECEPTOR-LIKE KINASE 902 associates with and phosphorylates BRASSINOSTEROIDSIGNALING KINASE1 to regulate plant immunity. Mol. Plant 12:59-70.

Zhou, J., Loh, Y. T., Bressan, R. A., and Martin, G. B. 1995. The tomato gene Ptil encodes a serine/threonine kinase that is phosphorylated by Pto and is involved in the hypersensitive response. Cell 83:925-935. 